\title{
Effects of ad libitum Ingestion of Monosodium Glutamate on Weight Gain in C57BL6/J Mice
}

\author{
Xueying Ren ${ }^{a, b}$ Jozélia G. Ferreira ${ }^{a, b}$ Catherine W. Yeckel ${ }^{a, c}$ Takashi Kondoh ${ }^{d}$ \\ Ivan E. de Araujo ${ }^{a, b}$ \\ ${ }^{a}$ The John B. Pierce Laboratory, Departments of ${ }^{b}$ Psychiatry and ${ }^{\mathrm{C}}$ Epidemiology and Public Health, Yale University \\ School of Medicine, New Haven, Conn., USA; ${ }^{d}$ Graduate School of Agriculture, University of Kyoto, Kyoto, Japan
}

\section{Key Words \\ C57BL6/J mice, weight gain - Indirect calorimetry · Low- and high-fat diets - Monosodium glutamate · Umami-tasting compounds}

\begin{abstract}
Although the umami compound monosodium glutamate (MSG) is a widely used flavor enhancer, controversy still persists regarding the effects of MSG intake on body weight. It has been claimed, in particular, that chronic MSG intake may result in excessive body weight gain and obesity. In this study we assessed the effects of chronic (16 weeks) ad libitum MSG on body weight and metabolism of C57BL6/J mice. Adult male mice were divided in four experimental groups and fed with either a low-fat (LF) or high-fat (HF) diet and with either two bottles of plain water or one bottle containing $1 \%$ MSG and another one containing water according to a factorial design. Mice were monitored weekly for body weight and food/fluid intake for 15 weeks. At the end of the experiments, the circulating levels of leptin, insulin, total protein, total cholesterol, triglyceride, blood urea nitrogen, and non-esterified fatty acids were also analyzed. Our results show that MSG intake did not influence body weight in either LF or HF groups. Interestingly, although animals overall displayed strong preferences for MSG against water, preferences were relatively higher in LF compared to HF group.
\end{abstract}

Consistent with the body weight data, while significant differences in leptin, insulin, total cholesterol, and non-esterified fatty acids were found between HF and LF groups, such an effect was not influenced by MSG intake. Finally, indirect calorimetry measurements revealed similar energy expenditure levels between animals being presented water only and MSG only. In summary, our data does not support the notion that ad libitum MSG intake should trigger the development of obesity or other metabolic abnormalities.

Copyright $\odot 2011$ S. Karger AG, Basel

There are five known basic tastes - sweet, sour, salty, and bitter - the most recently discovered is umami. Umami-tasting compounds can function as flavor enhancers, by adding a meaty or savory note to otherwise less palatable foods. The most closely studied flavor enhancer is monosodium L-glutamate (MSG), a sodium salt of the amino acid L-glutamate, considered to be a prototypical umami tastant. Salts of glutamic acid were first discovered in 1908 when K. Ikeda first characterized the unique taste of umami attributed to glutamic acid [1]. Although considerable knowledge has been gained in recent years regarding how umami taste produced physiological effects on oral and gut receptors [1], the effects of ad libitum MSG on body weight gain remain highly controversial. In fact, earlier studies on putative toxic effects of MSG

\section{KARGER}

Fax +4161306 1234

E-Mail karger@karger.ch

www.karger.com
(C) $2011 \mathrm{~S}$. Karger AG, Basel

$0012-2823 / 11 / 0835-0032 \$ 38.00 / 0$

Accessible online at:

www.karger.com/dig
Ivan E. de Araujo

The John B. Pierce Laboratory and Yale University School of Medicine

290 Congress Avenue, New Haven, CT 06519 (USA)

Tel. +1 203562 9901, ext. 204, Fax +1 2036244950

E-Mail IAraujo@jbpierce.org 
on the brain claimed that MSG administration increases body weight (and fat mass) [2-5], giving origin to the popular belief that MSG may be a hazardous food component. However, more recent studies show that MSG ingestion may actually have the ability to suppress weight gain, fat deposition, and plasma leptin levels in male rats [6]. Given the controversial nature of the topic, and the lack of similar studies in mice, we investigated the effect of ad libitum MSG intake on body weight gain and metabolic parameters over a period of 15 weeks, in adult C57BL6/J mice fed with either low- or high-fat pellets, and having free access to one sipper containing a $1 \%$ MSG solution and a second one containing water. Two corresponding control groups having access to water only were also assessed.

\section{Material and Methods}

\section{Experimental Design}

Thirty-six C57BL/6J mice (The Jackson Laboratory), approximately 8 weeks old, were fed either a low-fat (LF) diet (Research Diets D12450B, $10 \mathrm{kcal} \%$ fat, $20 \mathrm{kcal} \%$ protein and $70 \mathrm{kcal} \% \mathrm{car}-$ bohydrate) or a 'very high-fat' (HF) diet (Research Diets D12492, $60 \mathrm{kcal} \%$ fat, $20 \mathrm{kcal} \%$ protein and $20 \mathrm{kcal} \%$ carbohydrate) in their home cages. All mice were given free access to two identical drinking sippers, symmetrically positioned on the home cage. Water was placed on side A, and a 1\% MSG (Ajinomoto Co., Japan) solution was placed on side $B$ of the home cages of 9 animals having access to LF ('LF MSG' group). Note that A or B do not denote a specific (left or right) side of the cage, but just name one particular position that was balanced across animals and interchanged throughout the duration of the experiment. An equivalent $\mathrm{HF}$ MSG group also containing 9 animals was defined. Two additional control groups, containing 9 animals each, were also defined ('LF water' and 'HF water' groups); for these two groups, two drinking sippers containing water, placed on sides A and B, were presented. Body weight as well as the consumption of food, water and/ or MSG were monitored weekly for 15 weeks. All experiments were conducted in accordance with the John B. Pierce Laboratory and Yale University regulations on usage of animals in research.

Plasma Analysis

Hormones (insulin and leptin), total cholesterol, triglyceride (TG), blood urea nitrogen (BUN), total protein, and non-esterified fatty acids (NEFA) in the plasma of the mice were measured by the Yale Mouse Metabolic Phenotyping Center.

\section{Glucose and Glycogen Measurements}

Blood glucose was measured with a hand-held glucometer (OneTouch) and data presented as $\mathrm{mg} / \mathrm{dl}$. Liver glycogen was immediately hydrolyzed from $\sim 150 \mathrm{mg}$ of tissue using $0.6 \mathrm{ml} 2 \mathrm{~N} \mathrm{HCl}$ for $2 \mathrm{~h}$ at $100^{\circ} \mathrm{C}$. Measurement of the resulting glucose units was carried out on an YSI Automated Glucose Analyzer by glucose oxidase methods (Yellow Springs Instruments, Inc., Yellow Springs, Ohio, USA). Data are presented as glucosyl units in $\mu \mathrm{mol} / \mathrm{g}$ wet weight.

Effects of ad libitum Ingestion of MSG on Weight Gain in C57BL6/J Mice

\section{Indirect Calorimetry}

Energy expenditure was measured via indirect calorimetry using the Oxymax/CLAMS Animal Monitoring System (Columbus Instruments, Columbus, Ohio, USA). The mechanism and calculation of $\mathrm{VO}_{2}, \mathrm{VCO}_{2}, \mathrm{RQ}$ and heat are the same as described before [7].

\section{Results}

\section{Body Weight, Food and Fluid Intake, and MSG}

Preference

The initial body weights in the four groups (i.e. LFD water, LFD MSG, HF water and HFD MSG) were not significantly different between them. The body weight, food consumption and fluid intake were monitored every week. A two-way food type $\times$ drink type ANOVA model revealed a significant effect of food type $(p<0.001)$, but not of drink type $(p>0.3)$ or interaction $(p>0.7)$ on total weight gain after 15 weeks. In fact, LF animals gained, overall, significantly less weight than HF ones (11.63 \pm $2.10 \mathrm{~g}$ in the LF water group, $10.63 \pm 1.77 \mathrm{~g}$ in the LF MSG group, $26.98 \pm 1.35 \mathrm{~g}$ in the HF water group and $25.07 \pm 1.15 \mathrm{~g}$ in the HF MSG group) (see fig. 1a). However, no significant effect was detected associated with MSG intake; in fact, direct pairwise comparisons revealed no differences between either the LF water and LF MSG groups (paired-sample t test, $\mathrm{p}>0.3$ ) or the HF water and HF MSG groups (paired-sample t test, $\mathrm{p}>0.1$ ).

Next, and consistent with the above, a two-way food type $\times$ drink type ANOVA revealed significant main effects of food type $(p<0.01)$, but not of drink ( $p>0.5)$ type, or interaction ( $p>0.9)$, on total food and caloric intake. Although the LF diet was overall consumed at higher levels than the HF diet (LF water: $329.59 \pm 8.13 \mathrm{~g}$, LF MSG: $325.79 \pm 8.86 \mathrm{~g}$, HF water: $302.74 \pm 6.81 \mathrm{~g}$, HF MSG: $298.26 \pm 7.50$ g; see fig. $1 \mathrm{~b}$ ), lower caloric intake was associated with the LF groups compared to HF groups (LF water: $1,267.28 \pm 31.27 \mathrm{kcal}$, LF MSG: $1,252.653 \pm 34.08 \mathrm{kcal}, \mathrm{HF}$ water: $1,587.29 \pm 35.72 \mathrm{kcal}$, HF MSG: 1,563.79 $\pm 39.31 \mathrm{kcal}$; see fig. 1c).

In LF and HF MSG groups, the MSG intake on side B was significantly higher than their water intakes on side A. A two-way food type $\times$ sipper side ANOVA revealed no main effects of food type $(p>0.1)$, but a significant effect of sipper side ( $\mathrm{p}<0.001)$, and interaction $(\mathrm{p}<0.01)$, on total intake. In fact, paired two-sample $\mathrm{t}$ tests revealed that the LF MSG group drank significantly more MSG than the HF MSG group ( $p<0.05$ ) (see fig. 1d). Although two-way ANOVA analysis of the effect of side and food on LF and HF water groups showed that 
Fig. 1. Total weight gain, total food, caloric and fluid intake and the preference for $1 \%$ MSG. Experimental data are presented as mean \pm SEM across animals throughout the text. a Total weight gain. After 15 weeks of ad libitum ingestion of MSG. LF diet groups gained significantly less weight than HF diet (two-way ANOVA, $p<0.001$ for food type, $p>0.3$ for drink type, $p>0.7$ for interaction). b Total food intake. Significantly more LF diet was consumed than HF diet (two-way, food type $\times$ drink type, $\mathrm{p}<0.01$ for food type, $p>0.5$ for drink type, or interaction ( $p>0.9)$. c Total caloric intake. Significantly less caloric intake in the LF diet group than the HF diet group (two-way, food type $\times$ drink type, $\mathrm{p}<0.01$ for food type, $\mathrm{p}>0.5$ for drink type, interaction $\mathrm{p}>0.9$ ). d Total fluid intake in MSG group. Water and MSG were put on sides A and B in the housing cages of LF MSG and HF MSG group. The MSG intakes on side B were significantly higher than their water intakes on side A in two MSG groups (LF and HF) (two-way ANOVA, food type $\times$ side type, $p>0.1$ for food type, $p<0.001$ for side type, $\mathrm{p}<0.01$ for interaction). Paired-samples $t$ test analysis revealed that LF MSG group drank significantly more MSG and less water than HF MSG group ( $p<0.05)$. e Two water bottles, one on side $\mathrm{A}$, the other one on side $\mathrm{B}$, were placed on the LF water and HF water groups. The water intake on side B was significantly higher than the water intake on side A (two-way ANOVA, side and food effects, $\mathrm{p}<0.001$ for side type, $\mathrm{p}>0.6$ for food type and $\mathrm{p}>$ 0.1 for interaction), but MSG intake on side B in each MSG group was significantly higher than water intake on side B in the corresponding water group (paired-sample t test LF/HF water group water intake on side B paired with LF/HF MSG group MSG intake on side $\mathrm{B}, \mathrm{p}<0.05)$. See text for details.



Table 1. Plasma analysis

\begin{tabular}{lrrrr}
\hline Hormone and others & \multicolumn{1}{l}{ LF water } & \multicolumn{1}{l}{ LF MSG } & \multicolumn{1}{l}{ HF water } & HF MSG \\
\hline Leptin, pg/ml & $11,130.75 \pm 3,068.259$ & $10,172.45 \pm 2,646.667$ & $54,130.65 \pm 12,258.71$ & $44,388.38 \pm 9,502.223$ \\
Insulin, pg/ml & $1,198.446 \pm 212.7842$ & $1,249.084 \pm 177.3662$ & $4,103.579 \pm 690.6801$ & $3,728.723 \pm 469.9948$ \\
Total cholesterol, mg/dl & $177.5722 \pm 9.792318$ & $184.8822 \pm 8.978635$ & $243.155 \pm 10.66651$ & $219.785 \pm 16.37689$ \\
Triglycerides, mg/dl & $54.04778 \pm 6.605022$ & $63.39722 \pm 4.070457$ & $62.06111 \pm 4.299748$ & $58.33611 \pm 3.971915$ \\
Total protein, g/dl & $5.41916 \pm 0.045639$ & $5.509166 \pm 0.092037$ & $5.415556 \pm 0.082959$ & $5.379444 \pm 0.128794$ \\
BUN, mg/dl & $23.02 \pm 1.635252$ & $25.14333 \pm 1.633379$ & $20.61333 \pm 1.095495$ & $22.09444 \pm 1.242657$ \\
NEFA, mmol/l & $0.881667 \pm 0.085127$ & $0.806111 \pm 0.060195$ & $0.577778 \pm 0.036734$ & $0.585 \pm 0.066484$ \\
\hline
\end{tabular}

the intake on side $\mathrm{B}(172.16 \pm 13.71 \mathrm{ml}$ in $\mathrm{LF}$ water group, $152.26 \pm 8.70 \mathrm{ml}$ in $\mathrm{HF}$ water group) was significantly higher than that on side A $(110.69 \pm 11.99 \mathrm{ml}$ in $\mathrm{LF}$ water group, $122.39 \pm 5.74 \mathrm{ml}$ in $\mathrm{HF}$ water group) $(\mathrm{p}<0.001$ for side, $\mathrm{p}>0.6$ for food type and $\mathrm{p}>0.1$ for interaction), MSG intake on side B in each MSG group was significantly higher than water intake on side B in the corresponding water group (paired-sample t test LF/ $\mathrm{HF}$ water group water intake on side B paired with LF/ HF MSG group MSG intake on side $B, p<0.05$; see fig. 1e). Also, the time courses of the preferences in LF and HF groups make the differences in preference evi- dent (see fig. 1f): The preference for $1 \%$ MSG solution was relatively stable and always significantly higher than 0.5 within 15 weeks in LF MSG group (one-sample t test, $\mathrm{p}<0.001)$. The preference for MSG changed across time in the HF MSG group. After 8 weeks of MSG intake, the preference was no longer significantly different from 0.5 (one-sample $t$ test, $\mathrm{p}>0.05$ ). After a certain period of MSG ingestion in the HF diet group, mice decreased the intake of MSG. The overall preference for MSG was significantly higher in LF MSG group than that in HF MSG group $(86.27 \pm 0.61 \%$ in the LF MSG group, $65.53 \pm$ $2.06 \%$ in HF MSG group) ( $\mathrm{p}<0.05)$. 


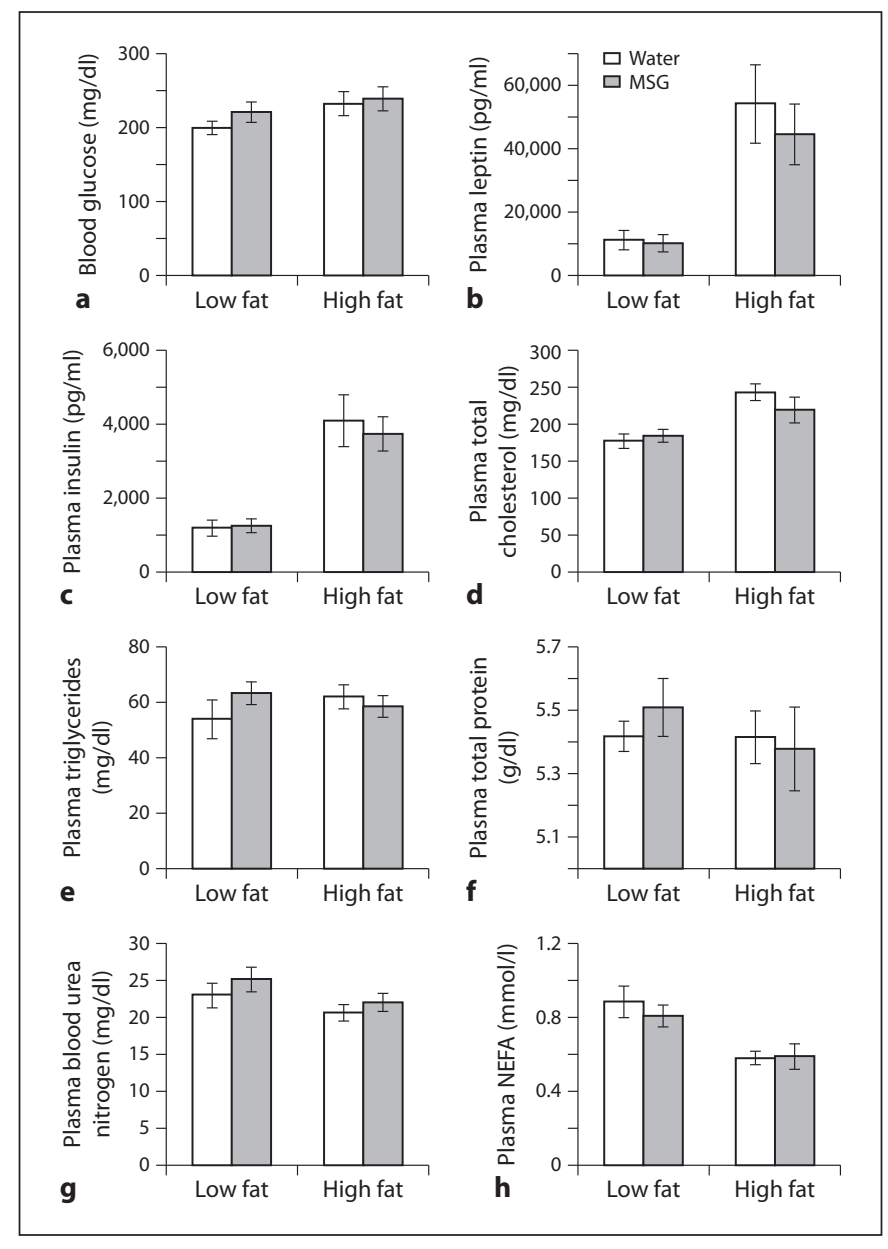

Fig. 2. Blood glucose and plasma analysis. a Blood glucose. Similar blood glucose was obtained among the four groups. b Leptin levels were significantly higher in HF than LF diet groups. c Insulin levels were significantly higher in HF than LF diet groups. d Total cholesterol levels were significantly higher in HF than LF diet groups. e Levels of plasma TGs were not significantly different between LF and HF diet groups. $\mathbf{f}$ Levels of plasma total protein were not significantly different between LF and HF diet groups. g Levels of plasma BUN were not significantly different between LF and HF diet groups. $\mathbf{h}$ Levels of plasma NEFA were significantly higher in LF than HF diet groups. Statistics for a, e, $\mathbf{f}$ and $\mathbf{g}$ are by two-way ANOVA, food and drink type, $\mathrm{p}>0.05$ for both factors and interaction. Statistics for $\mathbf{b}, \mathbf{c}, \mathbf{d}$ and $\mathbf{h}$ are by two-way ANOVA, $p<0.01$ for food type, $p>0.1$ for drink type and interaction. See text for details.

\section{Plasma Analysis}

Blood glucose was measured immediately after the mice were sacrificed and was not significantly different across the four experimental groups (two-way ANOVA, food and drink type, $\mathrm{p}>0.05$ for both factors and interaction). Plasma analysis revealed significant effects of

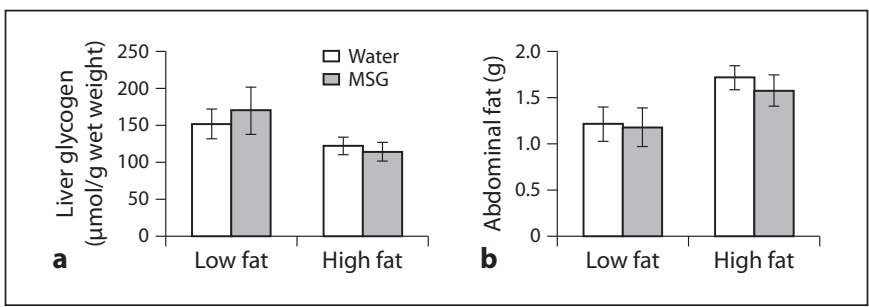

Fig. 3. Liver glycogen and abdominal fat analysis. a Levels of liver glycogen were significantly higher in LF than HF diet groups. b Amounts of abdominal fat were significantly higher in HF than LF diet groups. Two-way ANOVA was employed, food and drink type, $\mathrm{p}<0.05$ for food type, $\mathrm{p}>0.5$ for drink type and interaction.

food type on leptin, insulin, and total cholesterol plasma levels, which as expected were significantly higher in HF groups than that in LF groups. Conversely, NEFA was significantly lower in HF than LF groups, but this difference did not depend on drink type (two-way ANOVA, $p<0.01$ for food type, $p>0.1$ for drink type and interaction). No significant differences were found in the levels of TGs, total protein or BUN between any of the groups (see table 1, fig. 2).

\section{Liver Glycogen and Abdominal Fat Measurements}

Although significant differences were found in liver glycogen and abdominal fat between the LF and HF diet groups (two-way ANOVA, $\mathrm{p}<0.05$ for food type, but $\mathrm{p}>$ 0.5 for drink type and interaction), no significant differences were found between the water and MSG groups (see fig. 3a, b).

\section{Indirect Calorimetry}

To assess whether stronger concentrations of MSG could produce more robust effects on metabolism in C57BL6/J mice, mice were given a 10\% MSG solution, but no food, during indirect calorimetry measurements using metabolic cages. A significant difference was found in RER before and after water or 10\% MSG was given to the mice, but not significant between water or MSG group (two-way ANOVA, food and drink type, $\mathrm{p}<0.05$ for before and after the drink, $\mathrm{p}>0.7$ for drink type and interaction). The MSG intake was significantly larger than water during these indirect calorimetry sessions (one-way ANOVA, $\mathrm{p}<0.05$ ). No significant differences were found in $\mathrm{VO}_{2}, \mathrm{VCO}_{2}$ and heat between groups. Ambulatory activity was not significantly different within groups before or after water or MSG was supplied (repeated measure, $p>0.9)$ (see table 2). 
Table 2. Indirect calorimetry

\begin{tabular}{llll}
\hline Calorimetry & Group & Before & After \\
\hline $\mathrm{VO}_{2}, \mathrm{ml} / \mathrm{kg} / \mathrm{h}$ & Water & $1,887.13 \pm 308.27$ & $1,574.75 \pm 252.71$ \\
& MSG & $1,708.50 \pm 238.22$ & $1,735.00 \pm 329.64$ \\
\hline $\mathrm{VCO}_{2}, \mathrm{ml} / \mathrm{kg} / \mathrm{h}$ & Water & $1,392.13 \pm 230.63$ & $1,135.75 \pm 183.42$ \\
& MSG & $1,264.88 \pm 178.88$ & $1,241.25 \pm 230.15$ \\
\hline $\mathrm{RER}$ & Water & $0.73625 \pm 0.004317$ & $0.7215 \pm 0.005392$ \\
& MSG & $0.738875 \pm 0.002799$ & $0.722125 \pm 0.006934$ \\
\hline Heat, kcal/h & Water & $0.030059 \pm 0.001518$ & $0.024575 \pm 0.002341$ \\
& MSG & $0.028124 \pm 0.001721$ & $0.029375 \pm 0.00302$ \\
\hline Total licks & Water & Not available & $1,404.43 \pm 232.30$ \\
& MSG & Not available & $3,302.88 \pm 511.24$ \\
\hline Ambulatory & Water & 407.89 \pm 95.11 & $494.19 \pm 1.92$ \\
activity & MSG & $440.26 \pm 77.17$ & $477.38 \pm 71.04$ \\
\hline
\end{tabular}

\section{Conclusion and Discussion}

In this study, we have shown that in adult male C57BL6 mice, ad libitum 1\% MSG intake does not influence body weight gain patterns, independently of whether animals were fed an LF or HF diet. This was irrespective of the fact that, in the majority of the cases, the $1 \%$ MSG solution was greatly preferred over plain water. However, although food intake was not influenced by MSG, preference for $1 \%$ MSG against plain water was much higher in animals given an LF diet compared to an HF diet. Specifically, while mice fed LF diets and having access to MSG showed a robust and consistent preference for MSG, mice fed HF diets steadily reduced their preference for MSG after 8 weeks of the beginning of the experiment. Therefore, it seems that, instead, it is the food type that may influence MSG intake according to some currently undefined temporal patterns. Further research should investigate how
HF diets interact with behavioral preferences for MSG. In addition, analysis of plasma and liver glycogen did not show any important differences between MSG and water groups, although, as expected, these factors were significantly affected by food type. Finally, our indirect calorimetry measurements did not detect any differences between ad libitum water and 10\% MSG ingestion on metabolic parameters including $\mathrm{O}_{2}$ consumption, respiratory exchange ratios, and therefore energy expenditure levels.

Many investigators have been attracted by the intriguing question of how MSG may affect body weight and metabolism. Earlier studies claimed that MSG induces obesity through elevation of plasma glutamate concentrations, which would then penetrate into the brain, producing toxic effects on regions controlling energy homeostasis. However, recent studies have started to change this picture [6]. Plasma glutamate concentration was not affected when $1 \%$ MSG was ingested by rats, which actually showed weight gain reduction when having ad libitum access to MSG and HF diets [6]. Consistent with the above and our own data, a recent study shows that MSG is not associated with Chinese adult obesity or a greater prevalence of weight gain over 5 years [8]. However, further studies are needed to elucidate the mechanisms through which HF ingestion interferes with MSG palatability.

\section{Acknowledgements}

This study was supported by a Research Grant awarded by Ajinomoto Co., Japan. We greatly appreciate the discussions and intellectual input provided by K. Torii, E. Kimura as well as Drs Tsurugizawa and Ho (Ajinomoto Co., USA).

\section{Disclosure Statement}

No conflicts of interest to disclose.

\section{References}

$>1$ Jinap S, Hajeb P: Glutamate. Its applications in food and contribution to health. Appetite 2010;55:1-10.

$>2$ Hermanussen M, García AP, Sunder M, et al: Obesity, voracity, and short stature: the impact of glutamate on the regulation of appetite. Eur J Clin Nutr 2006;60:25-31.

$\checkmark 3$ Iwase M, Ichikawa K, Tashiro K, et al: Effects of monosodium glutamate-induced obesity in spontaneously hypertensive rats vs. $\mathrm{Wi}$ star-Kyoto rats: serum leptin and blood flow to brown adipose tissue. Hypertens Res 2000;23:503-510.
-4 Olney JW: Brain lesions, obesity, and other disturbances in mice treated with monosodium glutamate. Science 1969;164:719722 .

5 Tanaka K, Shimada M, Nakao K, Kusunoki T: Hypothalamic lesion induced by injection of monosodium glutamate in suckling period and subsequent development of obesity. Exp Neurol 1978;62:191-199.

6 Kondoh T, Torii K: MSG intake suppresses weight gain, fat deposition, and plasma leptin levels in male Sprague-Dawley rats. Physiol Behav 2008;95:135-144.
7 Ren X, Ferreira JG, Zhou L, et al: Nutrient selection in the absence of taste receptor signaling. J Neurosci 2010;30:8012-8023.

8 Shi Z, Luscombe-Marsh ND, Wittert GA, et al: Monosodium glutamate is not associated with obesity or a greater prevalence of weight gain over 5 years: findings from the Jiangsu Nutrition Study of Chinese adults. Br J Nutr 2010;104:457-463. 